\title{
Hacer cumplir las regulaciones sobre venta y consumo del alcohol como prevención ambiental universal
}

\section{Enforcing regulations on alcohol sales and use as universal environmental prevention}

\author{
Joan R. Villalbí*,**,***,****, Montserrat Bartroli*,***, Marina Bosque-Prous*,***,****, Anna \\ M Guitart*,***, Enric Serra-Batiste*****, Conrad Casas***, M. Teresa Brugal*,***
}

* Agència de Salut Pública de Barcelona. ** CIBER de Epidemiología y Salud Pública. *** Institut d'Investigacions Biomèdiques Sant Pau. **** Departament de Ciències Experimentals i de la Salut, Universitat Pompeu Fabra. ****** Direcció de Serveis de Llicències i Inspecció, Ajuntament de Barcelona

\section{Resumen}

El control social informal sobre el consumo de alcohol, tradicional en los países del Sur de Europa, se ha debilitado. Este cambio ha ido acompañado de un incremento de los episodios de consumo intensivo y borracheras en jóvenes en España. Para mitigar este problema, se han adoptado regulaciones sobre alcohol y conducción, y otras que restringen la venta y el consumo de alcohol. Este trabajo documenta las regulaciones vigentes y describe los esfuerzos realizados en la ciudad de Barcelona en este campo y sus resultados. Asimismo, se recopilan datos de infracciones denunciadas en los sistemas de información municipal para el período 2008-13.

Se observa un incremento de la presión de los servicios municipales para hacer cumplir las normas en dos aspectos: a) la venta en horario nocturno (establecimientos); y b) el consumo en la vía pública (ciudadanía). Por otra parte, también se aprecia un aumento en la actividad de control de la alcoholemia de los conductores, mientras que la proporción que superan los límites legales descendió. El mayor incremento relativo se ha producido en las acciones sobre establecimientos.

En Barcelona se realizan intervenciones para limitar la oferta y consumo de alcohol a bajo coste y en horario nocturno, así como la conducción bajo la influencia del alcohol. En la ciudad no se han documentado episodios de botellón masivo en espacios públicos. Estas acciones, que complementan otros esfuerzos preventivos basados en la educación para la salud, pueden modificar la percepción social del alcohol por los menores en un sentido menos favorable al consumo, contribuyendo a crear un entorno de prevención ambiental.

Palabras clave: alcohol, prevención, regulación, autoridad.

\begin{abstract}
The informal social control over alcohol consumption that was traditional in Southern European countries has weakened. At the same time there is an increase in binge drinking and drunkenness among young people in Spain. To mitigate this problem, regulations on alcohol and driving and restrictions on the sale and consumption of alcohol have been adopted. This paper documents the current regulations in the city of Barcelona and describes efforts to enforce them and their outcomes. Data from the municipal information systems on infringements reported for the period 2008-13 are provided.

There is an increasing pressure of municipal services to enforce the rules in two areas: a) alcohol sales at night (retailers); and b) consumption in the public space (citizens). An increase in the controls of drink-driving has also taken place, and the proportion above legal limits has decreased. The largest relative increase occurred in the control of retailers.

In Barcelona interventions are made to limit the supply and consumption of alcohol at low cost and during the night, and of driving under the influence of alcohol. There have been no documented episodes of massive drinking in public spaces (known as 'botellón') in the city. These actions, which complement other preventive efforts based on health education, can change the social perceptions of alcohol by minors in a direction less favorable to consumption, promoting environmental prevention. Key words: alcohol, prevention, regulation, authority.
\end{abstract}


D urante años se ha considerado que en los países del Sur de Europa el consumo de alcohol estaba integrado en la estructura cultural, sin mecanismos restrictivos de control formal, y con poca visibilidad del consumo problemático (Room y Mäkelä, 2000). Con los cambios sociales de las últimas décadas, este esquema se ha cuestionado (Anderson y Baumberg, 2006). Por un lado, hay un menor consumo medio y menos consumo diario, y por otro, se aprecia un incremento en los episodios de consumo intensivo y borracheras en jóvenes en nuestro país, con consecuencias sanitarias y sociales (Villalbí y Brugal, 2012). Esto refleja que en algunos segmentos juveniles hay una percepción del consumo intensivo como normativo entre sus pares (Calafat Far, 2007; Pascual Pastor, 2002; Salamó, Gras y Font-Mayolas, 2010), además de amplia accesibilidad al alcohol. Progresivamente, se han adoptado diversas regulaciones formales como respuesta a este problema (Villalbí, Bosque-Prous, Gili-Miner, Espelt y Brugal, 2014). Las más visibles y conocidas son las relativas al alcohol y la conducción, que han conseguido una importante reducción de las lesiones por tráfico en España (Pulido et al., 2014). Otras regulaciones relevantes son las que afectan a la disponibilidad del alcohol, mediante restricciones a su venta y consumo (Valencia-Martín, Galán y Rodríguez-Artalejo, 2007), que diversos gobiernos locales y autonómicos han adoptado y luego se han aplicado de forma variable según el contexto. El valor de las políticas regulatorias para reducir el consumo problemático de alcohol se ha revisado recientemente de forma exhaustiva (Babor et al., 2010).

La ciudad de Barcelona se ha dotado desde 1989 de planes de acción sobre las drogodependencias, incluyendo tanto las drogas ilícitas como las lícitas, poniendo especial énfasis en el alcohol. Estos planes se han aprobado en el Pleno Municipal tras buscar y conseguir consenso político (Manzanera et al., 2000). Son transversales e incorporan objetivos relacionados con diversos servicios municipales y sanitarios (Brugal, Guitart y Espelt, 2013). Su coordinación y evaluación se confiaron desde el inicio a los servicios de salud pública, actualmente concentrados en la Agència de Salut Pública de Barcelona (ASPB). Ésta es un organismo autónomo de tipo consorcial entre el gobierno local y el autonómico que agrupa todos los servicios de salud pública de la ciudad, y en el ámbito de los Planes de drogas actúa en coordinación con otros servicios municipales y también con el Departamento de Salud y el Servicio Catalán de la Salud (Guix et al., 2008). Como parte de esta labor, fomenta el cumplimiento efectivo de las regulaciones sobre la promoción, venta y consumo de alcohol en la ciudad, y recoge datos de las acciones coercitivas de los diversos servicios municipales en este ámbito.

El objetivo de este trabajo es documentar los esfuerzos realizados para la aplicación en la ciudad de las regulaciones vigentes relativas al alcohol. Se compila y analiza infor- mación que refleja las acciones desarrolladas en este campo y su evolución a lo largo del tiempo, discutiendo su aportación desde una perspectiva preventiva integrada.

\section{Método}

Este es un estudio descriptivo centrado en la ciudad de Barcelona durante el período de seis años de 2008-13. Se recopilan los datos de las principales categorías de denuncias por infracción de las normativas referidas al alcohol practicadas por la Guardia Urbana de Barcelona (denominación de la policía local en la ciudad) recogidos en los sistemas de información municipal sobre infracciones, denuncias y sanciones. Se describe su evolución a lo largo de estos años, y se comparan los datos del primer trienio (2008-10) con el segundo (2011-13), se valoran los cambios mediante la prueba de ji cuadrado para la comparación de proporciones o el cálculo del intervalo de confianza al 95\%, utilizando el programa epidat. Los sistemas de información de los que se extraen los datos de denuncias están centralizados en aplicativos informáticos municipales. Los datos agregados y anonimizados de infracciones denunciadas relacionadas con el alcohol se han obtenido por los servicios centrales de la Guardia Urbana que los facilitan a los servicios de salud pública como parte del seguimiento de los objetivos del Plan de Acción sobre Drogodependencias.

Las normativas relativas al alcohol en cuyo cumplimiento se implica la Guardia Urbana son básicamente tres, y se recogen en la tabla 1 . Todas ellas estaban en vigor antes de 2008, inicio del período estudiado. La normativa estatal de tráfico está recogida en la Ley sobre Tráfico, Circulación de Vehículos a Motor y Seguridad Vial, y sus sucesivas modificaciones (Real Decreto Legislativo 339/1990, de 2 de marzo, por el que se aprueba el Texto Articulado de la Ley sobre Tráfico, Circulación de Vehículos a Motor y Seguridad Vial, 1990). Ésta prohíbe la conducción si la alcoholemia supera determinados límites. Por encima de éstos, se aplican las sanciones administrativas correspondientes (retirada de puntos en el carné de conducir y multa, habitualmente de 500 euros).

La normativa autonómica sobre venta y consumo de alcohol incluida en la ley de prevención y asistencia en materia de sustancias que puedan generar dependencia de Cataluña y sus sucesivas modificaciones (Llei 20/1985, de 25 de juliol, de prevenció $i$ assistència en matèria de substàncies que poden generar dependència, 1985). Ésta prohíbe la venta en establecimientos donde no se permite el consumo (comercio alimentario) desde las $23 \mathrm{~h}$ a las $8 \mathrm{~h}$ de la mañana siguiente y obliga a la rotulación visible de esta norma desde 1998. Asimismo, prohíbe la venta de bebidas alcohólicas a los menores de 18 años desde el año 2002. Tanto las infracciones por venta a menores como por venta nocturna se consideran graves, por lo que se asocian a sanciones cuyo importe mínimo es de 3.000 euros que requiere la incoación de un expediente sancionador. 
Tabla 1. Algunos aspectos de las regulaciones vigentes sobre el alcohol referidos a su venta, la conducción de vehículos, y el consumo en la vía pública. Barcelona, 2008-13

\begin{tabular}{|c|c|c|c|c|c|c|}
\hline $\begin{array}{l}\text { Objeto de la } \\
\text { regulación }\end{array}$ & $\begin{array}{l}\text { Ámbito } \\
\text { territorial de } \\
\text { la regulación }\end{array}$ & $\begin{array}{l}\text { Forma de la } \\
\text { regulación }\end{array}$ & $\begin{array}{l}\text { Aspectos concretos } \\
\text { regulados }\end{array}$ & $\begin{array}{l}\text { Fecha de la } \\
\text { regulación }\end{array}$ & $\begin{array}{l}\text { Principales actores } \\
\text { que velan por su } \\
\text { cumplimiento }\end{array}$ & $\begin{array}{l}\text { Infracciones y } \\
\text { sanciones }\end{array}$ \\
\hline \multirow{2}{*}{$\begin{array}{l}\text { Conducción de } \\
\text { vehículos bajo } \\
\text { los efectos del } \\
\text { alcohol }\end{array}$} & \multirow[t]{2}{*}{ España } & \multirow{2}{*}{$\begin{array}{l}\text { Ley sobre tráfico, } \\
\text { circulación de } \\
\text { vehículos a motor } \\
\text { y seguridad vial } \\
\text { (RDL 339/1990) }\end{array}$} & $\begin{array}{l}\text { Cifras de alcohol en aire } \\
\text { espirado y conducción } \\
\text { superiores a } 0,25 \mathrm{mg} / \mathrm{L} \\
\text { de aire espirado }(0,15 \text { en } \\
\text { conductores noveles y } \\
\text { profesionales). }\end{array}$ & $\begin{array}{l}1990, \\
\text { enmendada en } \\
2006\end{array}$ & $\begin{array}{l}\text { Guardia urbana y } \\
\text { Policía autonómica }\end{array}$ & $\begin{array}{l}\text { Falta muy grave } \\
\text { ( } 500 €) \text { y pérdida } \\
\text { de puntos en el } \\
\text { carné. }\end{array}$ \\
\hline & & & $\begin{array}{l}\text { Cifras de alcohol } \\
\text { superiores a } 0,6 \mathrm{mg} / \mathrm{L} \text { de } \\
\text { aire espirado }\end{array}$ & $\begin{array}{l}2007 \text { (enmiendas } \\
\text { a la Ley) }\end{array}$ & $\begin{array}{l}\text { Guardia urbana y } \\
\text { Policía autonómica }\end{array}$ & $\begin{array}{l}\text { Delito contra } \\
\text { la seguridad } \\
\text { del tráfico con } \\
\text { sanción penal }\end{array}$ \\
\hline \multirow[t]{2}{*}{ Venta de alcohol } & \multirow[t]{2}{*}{ Catalunya } & \multirow{2}{*}{$\begin{array}{l}\text { Ley de prevención } \\
\text { y asistencia } \\
\text { en materia de } \\
\text { sustancias que } \\
\text { puedan generar } \\
\text { dependencia (Ley } \\
\text { 20/1985) }\end{array}$} & $\begin{array}{l}\text { Prohibida venta en } \\
\text { establecimientos donde } \\
\text { no se permite el consumo } \\
\text { entre las } 23 \mathrm{~h} \text { y las } 8 \mathrm{~h} \text { de la } \\
\text { mañana. }\end{array}$ & $\begin{array}{l}1998 \text { (enmiendas } \\
\text { a la Ley) }\end{array}$ & $\begin{array}{l}\text { Guardia urbana y } \\
\text { Policía autonómica }\end{array}$ & $\begin{array}{l}\text { Falta grave } \\
(3.000 €)\end{array}$ \\
\hline & & & $\begin{array}{l}\text { Prohibida la venta a } \\
\text { menores de } 18 \text { años. }\end{array}$ & $\begin{array}{l}2002 \text { (enmiendas } \\
\text { a la Ley) }\end{array}$ & $\begin{array}{l}\text { Guardia urbana y } \\
\text { Policía autonómica }\end{array}$ & $\begin{array}{l}\text { Falta grave } \\
(3.000 €)\end{array}$ \\
\hline $\begin{array}{l}\text { Consumo de } \\
\text { alcohol en la vía } \\
\text { pública }\end{array}$ & $\begin{array}{l}\text { Barcelona } \\
\text { ciudad }\end{array}$ & $\begin{array}{l}\text { Ordenanza } \\
\text { Municipal de } \\
\text { medidas para } \\
\text { fomentar y } \\
\text { garantizar la } \\
\text { convivencia } \\
\text { ciudadana en el } \\
\text { espacio público }\end{array}$ & $\begin{array}{l}\text { Prohibido el consumo de } \\
\text { alcoholen la vía pública } \\
\text { que cause molestias, y } \\
\text { en envases de vidrio o } \\
\text { lata (fuera de terrazas de } \\
\text { establecimientos). }\end{array}$ & 2006 & Guardia urbana & Falta leve $(30 €)$ \\
\hline
\end{tabular}

La Ordenanza Municipal de medidas para fomentar y garantizar la convivencia ciudadana en el espacio público (Ordenanza de medidas para fomentar y garantizar la convivencia ciudadana en el espacio público de Barcelona, 2005), popularmente conocida como ordenanza del civismo, entró en vigor durante el año 2006. En ella se recogen dos prohibiciones relevantes: el consumo de alcohol en la vía pública cuando pueda causar molestias a vecinos o transeúntes, y su consumo en envases de vidrio o de lata, salvo en terrazas de bares y restaurantes. La infracción de estos preceptos tiene carácter leve, por lo que estas denuncias comportan una sanción de un importe mínimo de 30 euros, con la posibilidad de su sustitución por medidas alternativas.

\section{Resultados}

En la figura 1 se presenta el número de controles de alcoholemia practicados por la Guardia Urbana en conductores de vehículos de 2008 a 2013, y la proporción de pruebas que superan los límites establecidos. El número de controles parece estable, aunque con oscilaciones: la media es de unos 129.000 al año, y los valores muestran una amplitud de 115.023 a 142.336. En el período 2011-13 se realizó un 1,2\% más de controles que en 2008-10. En estos controles se generaron entre 9.000 y 11.000 denuncias al año por superar los límites marcados de alcohol en aire espirado. En el período 2008-10, el 8,11\% (IC 95\%: 8,03-8,20) de los controles superan el límite, mientras que en 2011-13 lo hace el 7,71\% (IC 95\%: 7,63-7,80), una proporción inferior.

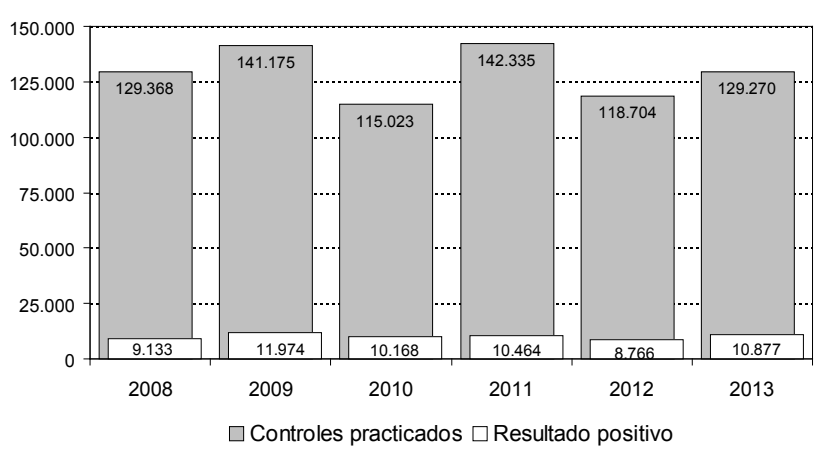

Figura 1. Controles de alcoholemia practicados por la Guardia Urbana en conductores de vehículos y resultados que superan los límites establecidos. Barcelona, 2008-13.

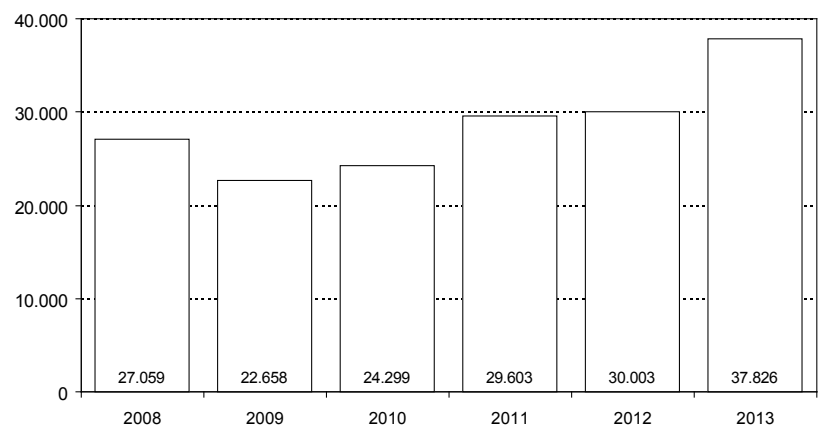

Figura 2. Denuncias de la Guardia Urbana por consumo de alcohol en la vía pública contraviniendo las ordenanzas municipales. Barcelona, 2008-13. 


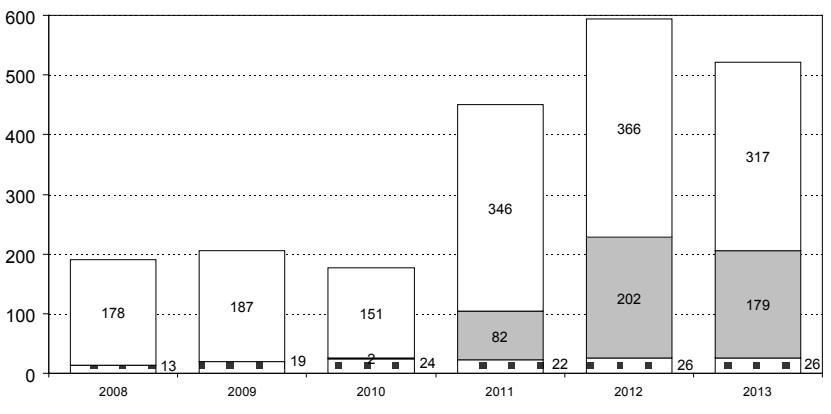

$\square$ Venta a menores $\square$ Venta en horario nocturno $\square$ Otras infracciones

Figura 3. Denuncias de la Guardia Urbana a establecimientos por infracciones de las normas referidas a venta de alcohol, por tipo de infracción. Barcelona, 2008-13.

La figura 2 muestra las denuncias practicadas por la Guardia Urbana por consumo de alcohol en la vía pública. Tras la entrada en vigor de la ordenanza municipal, la cifra anual de denuncias se ha incrementado, pasando de unas 27.000 en 2008 a aproximadamente 38.000 en 2013. En términos de trienios, pasan de 74.016 a 97.432 denuncias, que en tasas anuales serían 1.759,77 (IC 95\%: 1.747,12-1.772,50) y 2.316,50 (IC 95\%: 2.301,98-2.331,09) sanciones por 100.000 habitantes mayores de 14 años -año.

La figura 3 ilustra el número de denuncias de la Guardia Urbana a establecimientos de pública concurrencia (principalmente comercios alimentarios, bares y cafeterías) por infracciones relacionadas con el alcohol entre 2008 y 2013. Se detallan las causadas por la venta de bebidas alcohólicas a menores, las debidas a la venta de alcohol durante el horario nocturno, y las relativas a otras infracciones, la mayoría por falta de rotulación. Como puede apreciarse, a partir de 2011 el número de denuncias se incrementó notablemente, destacando la emergencia de denuncias relacionadas con la venta en horario nocturno. Éstas pasan de ser testimoniales y limitadas a alguna gasolinera situada dentro del casco urbano a unas 200 denuncias al año, y de representar el $0,35 \%$ de las denuncias en el primer trienio a representar el $29,57 \%$ en el segundo $(\mathrm{p}<0,0001)$. Las denuncias por venta a menores se duplicaron, de 13 a 26 al año, pero representan un menor porcentaje del total de denuncias, bajando del $9,76 \%$ al $4,73 \%(\mathrm{p}<0,0001)$.

\section{Discusión}

Aunque en los medios de comunicación y en internet aparecen con frecuencia informaciones sobre sanciones a locales o ciudadanos relacionadas con la venta o el consumo de alcohol, no hemos encontrado datos de otras ciudades o comunidades sobre su frecuencia absoluta o relativa; tan sólo los datos relacionados con la seguridad vial aparecen recogidos sistemáticamente en informes de la Dirección General de Tráfico (DGT) o las policías autonómicas. Los datos de Barcelona sugieren que, en la ciudad, las normas legales surgidas en los últimos años se están traduciendo en un esfuerzo organizado y cada vez más coherente orientado a reducir la oferta y consumo de alcohol a bajo coste accesible a menores y en horario nocturno y la conducción bajo la influencia del alcohol. De acuerdo con los esquemas propuestos por Foxcroft (2014), estas acciones, que complementan otros esfuerzos preventivos basados en la educación para la salud, deberían modificar la percepción social del consumo de alcohol en los menores en un sentido menos favorable al consumo, contribuyendo a crear un entorno de prevención ambiental. Quizás en el futuro se traduzca en diferencias en el patrón de consumo adolescente en la ciudad con respecto a otros ámbitos. En todo caso, no se han documentado en la ciudad episodios de botellón masivo en espacios públicos, que sí se han producido en otras ciudades de nuestro medio (Rodríguez-Martos, 2007).

Se puede intentar una aproximación a la magnitud relativa de estos esfuerzos, que será clave para su percepción pública. Se estima que en Barcelona hay 968.000 vehículos censados, además de los que circulan en la ciudad procedentes del ámbito metropolitano (Departament d'estadística. Ajuntament de Barcelona, 2013). Esto permite estimar que hay 13 controles de alcoholemia al año por cada 100 vehículos censados. Los controles se realizan con más frecuencia en horario nocturno, en fin de semana, y en zonas con más locales de ocio, y se detecta una infracción por cada 13 controles realizados. Las actuaciones de la Guardia Urbana se suman a las que realiza el Cuerpo de Mossos de Esquadra: entre 400.000 y 600.000 controles al año en Catalunya, especialmente en vías interurbanas. En su conjunto, configura una presión notable respecto al control de la conducción bajo los efectos del alcohol, que ha mostrado un impacto favorable en los indicadores de alcoholemia basados en muestras representativas y en las lesiones por tráfico (Alcañiz et al., 2014; Pulido et al., 2014; Sarasa-Renedo et al., 2014). Por lo que respecta a los establecimientos minoristas de alimentación, se estima que hay 4.200 en la ciudad, la mayoría de los cuales cierra antes de las 21h. Si los usamos como denominador, las denuncias por venta en horario nocturno en este sector serían unas 5 por cada 100 establecimientos en el último año estudiado. No podemos estimarla con respecto a los que abren en horario nocturno por no tener cifras de referencia, pero es de suponer que sería muy superior. Estas estimaciones de frecuencia relativa, como las realizadas para las denuncias por consumo en la vía pública, no son realmente tasas (pues requerirían de asunciones sobre numeradores y denominadores que no se cumplen), pero son muy orientativas.

Esta dinámica debe ponerse en el contexto de los cambios que se han dado en el mercado de la venta de bebidas alcohólicas y en la oferta de ocio en la ciudad. Por un lado, la creciente desregulación de horarios comerciales ha supuesto que la venta de alcohol en los establecimientos alimentarios (tradicional en nuestro medio y no sometida a licencia) se haya ampliado durante la noche y los fines de semana. La limitación de venta en horario nocturno intenta precisamen- 
te paliar los problemas que el acceso a bebidas alcohólicas a bajo coste comporta (Villalbí et al., 2014). Por otro lado, el sector de hostelería se ha ampliado notablemente en una ciudad en la que el turismo tiene un peso cada vez mayor en la economía (Barcelona Turisme, 2014; Departament d'estadística. Ajuntament de Barcelona, 2014). Finalmente, los cambios en la dinámica familiar y los patrones de ocio adolescente y juvenil llevan a que la proporción de jóvenes (incluso de menores) fuera de su casa hasta horas avanzadas de la noche haya aumentado, y esto se asocia con el consumo de riesgo (Llorens, Barrio, Sánchez, Suelves y ESTUDES Working Group, 2011). Por tanto, el esfuerzo regulatorio y de velar por el cumplimiento de las regulaciones se contrapone a otros cambios sociales que favorecen un mayor consumo. Otra fuerza a tener en cuenta es el marketing de la industria del alcohol, del que hay indicios de su impacto en el consumo (Montes-Santiago, Alvarez Muñiz y Baz Lomba, 2007).

Evidentemente, hay que tener en cuenta otras acciones de los servicios de salud pública para reducir el consumo de riesgo de alcohol en los adolescentes y jóvenes. En la ciudad, destacan los programas educativos de prevención universal (Foxcroft y Tsertsvadze, 2012) ofrecidos en la educación secundaria y que alcanzan una cobertura relevante (Juárez, Pasarín y Arcas, 2014), así como las acciones de prevención selectiva basadas en intervenciones de prevención inespecífica en barrios considerados de mayor riesgo (Bartroli et al., 2012; Bartroli, Espelt, Castellano y Brugal, 2012), además de los programas de prevención indicada (Guitart et al., 2012). Pero como bien muestran las revisiones de la literatura, las acciones de prevención poblacionales tienen el máximo potencial preventivo (Martineau, Tyner, Lorenc, Petticrew y Lock, 2013). Habría que contemplar las acciones de ejercicio de la autoridad para hacer cumplir las normas formales relacionadas con la venta y consumo de alcohol en nuestro medio como acciones de prevención ambiental universal, que modifican el entorno (Burkhart, 2011; Foxcroft, 2014). La secuencia que va desde la aprobación de normas sobre el consumo de alcohol hasta su cumplimiento efectivo es clave para reducir las percepciones sociales que han llevado a la banalización del consumo de alcohol en algunos grupos de población. Este factor influye en el balance entre costes y beneficios del consumo percibidos por los jóvenes, así como en la norma social percibida (Vries, Backbier, Kok y Djkstra, 1995), que son determinantes clave de la conducta.

\section{Conflicto de intereses}

Los autores no declaran conflicto de intereses.

\section{Reconocimientos}

Los autores expresan su agradecimiento a los responsables de Sistemas de Información de la Guardia Urbana de Barcelona por facilitar los datos sobre denuncias. Este traba- jo se ha beneficiado de la financiación parcial de las Redes de investigación cooperativa (Red trastornos adictivos RTA) RD06/0001/1018 y RD12/0028/0018.

\section{Referencias}

Alcañiz, M., Guillén, M., Santolino, M., Sánchez-Moscona, D., Llatje, O. y Ramon, L. (2014). Prevalence of alcohol-impaired drivers based on random breath tests in a roadside survey in Catalonia (Spain). Accident Analysis and Prevention, 65, 131-141. doi:10.1016/j. aap.2013.12.021

Anderson, P y Baumberg, B. (2006). Alcohol in Europe. A public health perspective. London: Institute of Alcohol Studies.

Babor, T. F., Caetano, R., Casswell, S., Edwards, G., Giesbrecht, N., Graham, K., ... Rossow, I. (2010). Alcohol: No Ordinary Commodity: Research and Public Policy. New York: Oxford University Press.

Barcelona Turisme. (2014). Estadístiques de turisme a Barcelona $i$ comarques. Barcelona: Diputació de Barcelona y Barcelona Turisme.

Bartroli, M., Espelt, A., Castellano, Y. y Brugal, M. (2012). De marxa fent esport: perfil dels participants a la 1a fase del programa el curs 2010-11. Barcelona: Agència de Salut Pública de Barcelona.

Bartroli, M., Petit, M., Herrera, F., Vazquez, A., Espelt, A. y Brugal, M. (2012). La Karpa, un programa de prevención selectiva para promover un ocio saludable (p. 221). Presented at the XXXIX Jornadas Nacionales Socidrogalcohol, Tarragona: Socidrogalcohol. Recuperado de http://www.socidrogalcohol2012.org/documentos/ posteres/poster110.pdf

Brugal, M., Guitart, A. y Espelt, A. (2013). Pla d'Acció Sobre Drogues de Barcelona 2013-16. Barcelona: Agència de Salut Pública de Barcelona.

Burkhart, G. (2011). Environmental drug prevention in the EU. Why is it so unpopular? Adicciones, 23, 87-100.

Calafat Far, A. (2007). El abuso de alcohol de los jóvenes en España. Adicciones, 19, 217-223.

Departament d'estadística. Ajuntament de Barcelona. (2013). Transport i mobilitat. Recuperado de http:// www.bcn.cat/estadistica/catala/dades/anuari/cap15/ index.htm

Departament d'estadística. Ajuntament de Barcelona. (2014). Estadístiques econòmiques. Recuperado de http://www.bcn.cat/estadistica/catala/dades/econo$\mathrm{mia} /$ dirce/evo/t24.htm

Foxcroft, D. R. (2014). "Form ever follows function. This is the law". A prevention taxonomy based on a functional typology. Adicciones, 26, 10-14.

Foxcroft, D. R. y Tsertsvadze, A. (2012). Universal alcohol misuse prevention programmes for children and adolescents: Cochrane systematic reviews. Perspectives in Public Health, 132, 128-134. doi:10.1177/1757913912443487 
Guitart, A. M., Bartroli, M., Villalbi, J. R., Guilañá, E., Castellano, Y., Espelt, A. y Brugal, M. T. (2012). Prevención indicada del consumo problemático de drogas en adolescentes de Barcelona. Revista Española de Salud Pública, 86, 189-198.

Guix, J., Villalbí, J. R., Armengol, R., Llebaria, X., Manzanera, R., Plasència, A. y Comité de Dirección de la Agència de Salut Pública de Barcelona. (2008). Innovar en la gestión de servicios de salud pública: la experiencia de la Agencia de Salud Pública de Barcelona. Gaceta Sanitaria, 22, 267-274.

Juarez, O., Pasarin, M. y Arcas, M. (2014). Programes preventius en centres d'educació secundària de Barcelona. Avaluació del curs 2011-2012. Barcelona: Agència de Salut Pública de Barcelona.

Llei 20/1985, de 25 de juliol, de prevenció i assistència en matèria de substàncies que poden generar dependència (1985). Recuperado de http://portaljuridic.gencat. cat/ca/pjur_ocults/pjur_resultats_fitxa/?action=fitxa\&documentId=241334\&language $=$ ca_ES\&searchI$\mathrm{d}=4168506 \&$ mode $=$ single

Llorens, N., Barrio, G., Sánchez, A., Suelves, J. M. y ESTUDES Working Group. (2011). Effects of socialization and family factors on adolescent excessive drinking in Spain. Prevention Science, 12, 150-161. doi:10.1007/s11121-0100195-0

Manzanera, R., Torralba, L., Brugal, M., Armengol, R., Solanes, P. y Villalbi, J. R. (2000). Afrontar los estragos de la heroína: evaluación de diez años de un programa integral en Barcelona. Gaceta Sanitaria, 14, 58-66.

Martineau, F., Tyner, E., Lorenc, T., Petticrew, M. y Lock, K. (2013). Population-level interventions to reduce alcohol-related harm: an overview of systematic reviews. Preventive Medicine, 57, 278-296. doi:10.1016/j.ypmed.2013.06.019

Montes-Santiago, J., Alvarez Muñiz, M. L. y Baz Lomba, A. (2007). [Alcohol advertising in written mass media in Spain]. Anales de Medicina Interna (Madrid), 24, 109-112.

Ordenanza de medidas para fomentar y garantizar la convivencia ciudadana en el espacio público de Barcelona (2005). Recuperado de http://w110.bcn.cat/fitxers/ ajuntament/consolidadescast/convivencia.830.pdf

Pascual Pastor, F. (2002). Percepción del alcohol entre los jóvenes. Adicciones, 14 (Supl.1), 123-131.

Pulido, J., Indave-Ruiz, B. I., Colell-Ortega, E., Ruiz-García, M., Bartroli, M. y Barrio, G. (2014). Estudios poblacionales en España sobre daños relacionados con el consumo de alcohol. Revista Española de Salud Pública, 88, 493-513. doi:10.4321/S1135-57272014000400005

Real Decreto Legislativo 339/1990, de 2 de marzo, por el que se aprueba el Texto Articulado de la Ley sobre Tráfico, Circulación de Vehículos a Motor y Seguridad Vial (1990). Recuperado de http://noticias.juridicas.com/ base_datos/Admin/rdleg339-1990.html
Rodríguez-Martos, A. (2007). ¿Por qué es tan difícil legislar sobre alcohol en España? Adicciones, 19, 325-331.

Room, R. y Mäkelä, K. (2000). Typologies of the cultural position of drinking. Journal of Studies on Alcohol, 61, $475-483$.

Salamó, A., Gras, M. E. y Font-Mayolas, S. (2010). Patrones de consumo de alcohol en la adolescencia. Psicothema, 22, 189-195.

Sarasa-Renedo, A., Sordo, L., Molist, G., Hoyos, J., Guitart, A. M. y Barrio, G. (2014). Principales daños sanitarios y sociales relacionados con el consumo de alcohol. Revista Española de Salud Pública, 88, 469-491. doi:10.4321/ S1135-57272014000400004

Valencia-Martín, J. L., Galán, I. y Rodríguez-Artalejo, F. (2007). Binge drinking in Madrid, Spain. Alcoholism, Clinical and Experimental Research, 31, 1723-1730. doi:10.1111/j.1530-0277.2007.00473.x

Villalbí, J. y Brugal, M. (2012). Epidemiología del consumo de alcohol y de sus consecuencias en la salud. In F. Pascual Pastor \& J. Guardia Serecigni, Monografía sobre el alcoholismo (pp. 43-74). Barcelona: Socidrogalcohol.

Villalbí, J. R., Bosque-Prous, M., Gili-Miner, M., Espelt, A. y Brugal, M. T. (2014). Políticas para prevenir los daños causados por el alcohol. Revista Española de Salud Pública, 88, 515-528. doi:10.4321/S1135-57272014000400006

Vries, H., Backbier, E., Kok, G. y Djkstra, M. (1995). The Impact of Social Influences in the Context of Attitude, Self-Efficacy, Intention, and Previous Behavior as Predictors of Smoking Onset. Journal of Applied Social Psychology, 25, 237-257. 